\title{
Effects of Doping on the Morphology and Infrared Radiative Properties of Black Silicon
}

Sreyash Sarkar ${ }^{1 *}$, Ahmed A. Elsayed ${ }^{1,2}$, Frédéric Marty ${ }^{1}$, Jérémie Drévillon ${ }^{3}$, Yasser M. Sabry², Jiancun Zhao ${ }^{4}$, Yiting Yu $^{4}$, Elodie Richalot ${ }^{1}$, Philippe Basset ${ }^{1}$, Tarik Bourouina ${ }^{1}$, and Elyes Nefzaoui ${ }^{1}$

${ }^{1}$ Université Paris-Est, ESYCOM (FRE2028), CNAM, CNRS, ESIEE Paris, Université Paris-Est Marne-la-Vallée, F-77454

Marne-la-Vallée, France

${ }^{2}$ Electronics and Electrical Com. Depart., Faculty of Eng., Ain Shams University, Cairo, Egypt

${ }^{3}$ Institut Pprime, CNRS, Université de Poitiers, ISAE-ENSMA, Futuroscope Chasseneuil, France

${ }^{4}$ Northwestern Polytechnical University, Xi'an, China

* Corresponding Author: sreyash.sarkar@esiee.fr, +33605749505

\section{Abstract}

For the first time, we show that the density of nanostructures on Black Silicon obtained by wafer-level cryogenic plasma processing increases with a high level of doping, extending the spectral range of its very high absorptivity from near-infrared to far-infrared. We have found experimentally and confirmed by simulations that, for highly doped Black Silicon, a high absorptivity is observed till $15 \mu \mathrm{m}$. Subsequent processing of SEM images reveals that these noteworthy radiative properties are probably due to particular morphological features of heavily doped Black Silicon at the nano-scale. These features are quantified through statistical image processing. Reported results pave the way to highly integrated and effective infrared sources using Black Silicon.

\section{Introduction}

Radiative properties engineering is of paramount importance for several applications involving thermal radiation conversion and management such as thermophotovoltaics, thermal rectification, thermal memories, logical circuits and radiative cooling, consequently, drawing in an increased attention in recent years [1][2]. In the present work, we report, on the properties of Black Silicon (BSi) in the spectral range of thermal radiation, up to $25 \mu \mathrm{m}$ which can be instrumental for heat transfer applications. Black Silicon (BSi) has become nowadays, a well-established micro-nano-structured silicon surface that can be obtained by different techniques including cryogenic plasma [2] as considered in this work. BSi exhibits fascinating wetting and optical properties. In particular, due to its specific morphology, it is well-known for its excellent absorption of almost $100 \%$ of incident light [3][4][5], hence its name is Black according to its colour to the naked eye [6]. But very little is known about its infrared radiative properties and the possible ways of enhancing them for specific applications. It has been noted since $[7,8]$ that such nano-scale features lead to a critical improvement of silicon absorptivity in the visible range [9]. We show that we can extend such outstanding properties to the mid-infrared (MIR) by using highly doped silicon. We observe that the density of nanostructures on $\mathrm{BSi}$ increases with a high level of doping which leads to the increase of absorptivity over an extended spectral range. We have found experimentally and confirmed by simulations that, for highly doped BSi, high absorptivity is observed till $15 \mu \mathrm{m}$. Subsequent processing of SEM images reveals that these conspicuous radiative properties are presumably due to very specific morphological features of heavily doped BSi at the nano-scale. These features are quantified through statistical image processing. The promulgated outcomes, therefore, prepare the way to highly integrated infrared sources and promising performances in thermal management applications such as passive radiative cooling [10].

\section{Methodology}

We have fabricated two wafers of BSi having n-type doping of $2 \times 10^{18} \mathrm{~cm}^{-3}$ (standard doping) and $4.5 \times 10^{19} \mathrm{~cm}^{-3}$ (high doping). From top- and side-view SEM pictures, the mean values of periodicity and height of the BSi structures have been extracted by grey-level analysis [11] using MATLAB: all the centres of holes have been identified and each pixel corresponds to a height. Based on obtained data, simplified representative models of $\mathrm{BSi}$ are built to be used as inputs for electromagnetic simulations for radiative properties calculation based on FEM and Rigorous Coupled Wave Analysis (RCWA) [12] methods.

\subsection{Experimental Setup for Measurements of Reflectance}

Experimental measurements of specular reflectivity have been performed using a Perkin Elmer Spectrum GX FTIR Spectrometer in a spectral range from 1 to $25 \mu \mathrm{m}$ at room temperature and at different angles of incidences. Simulations and measurements on flat silicon with standard and high doping have also been performed for comparison.

\subsection{Black Silicon Fabrication}

A few methodologies have been created to limit the reflectance of silicon by texturing the surface $[13,14,15]$. The systems of silicon finishing by reactive ion etching (RIE) [16] and inductively coupled plasma-reactive ion etching (ICP-RIE), otherwise called deep reactive ion etching 


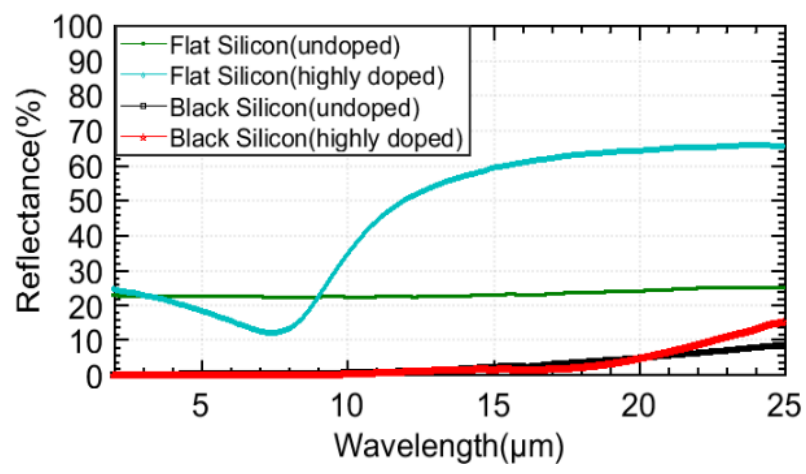

Figure 1: Measurements of reflectance on two BSi samples having standard and high doping at $30^{\circ}$ angle of incidence compared with flat Si. The highly doped BSi has a significantly higher absorption than the one with standard doping and the performance is evidently, better than highly doped flat Si.

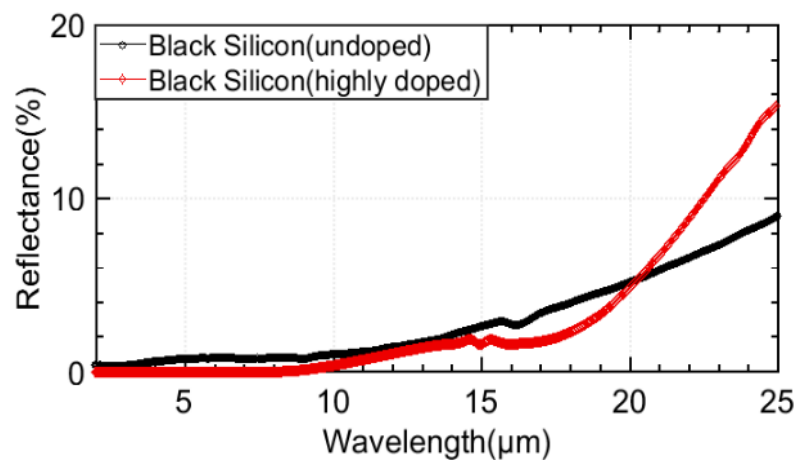

Figure 2: Zoom on the performance of highly doped and undoped black silicon further illustrating the fact that till $15 \mu \mathrm{m}$, highly doped sample has lower reflectance.

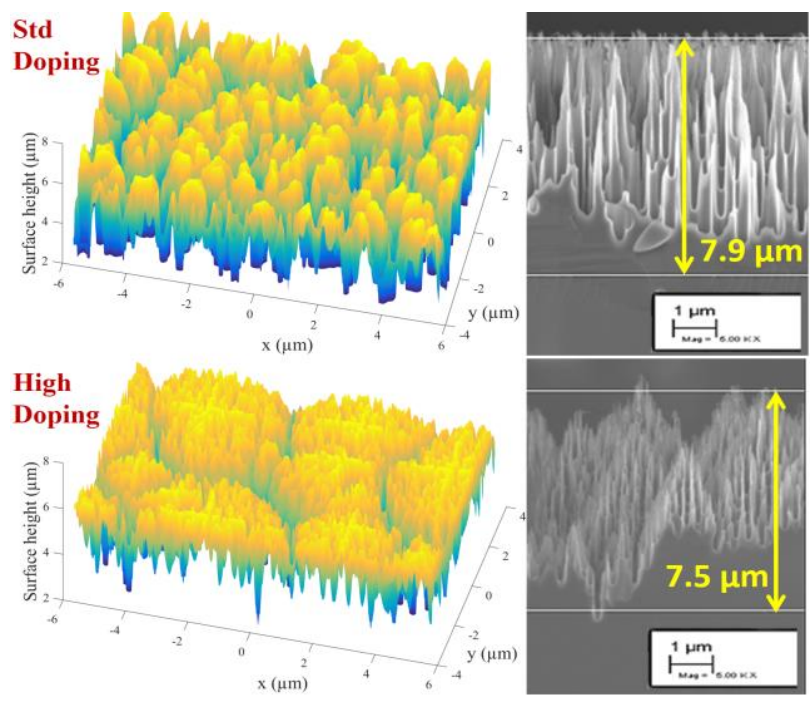

Figure 3: 3D reconstruction using gray-level technique from SEM images and side-view SEM for standard and highly doped silicon for a similar etching process: highlevel doping results in the increase of the density of nanostructurations.
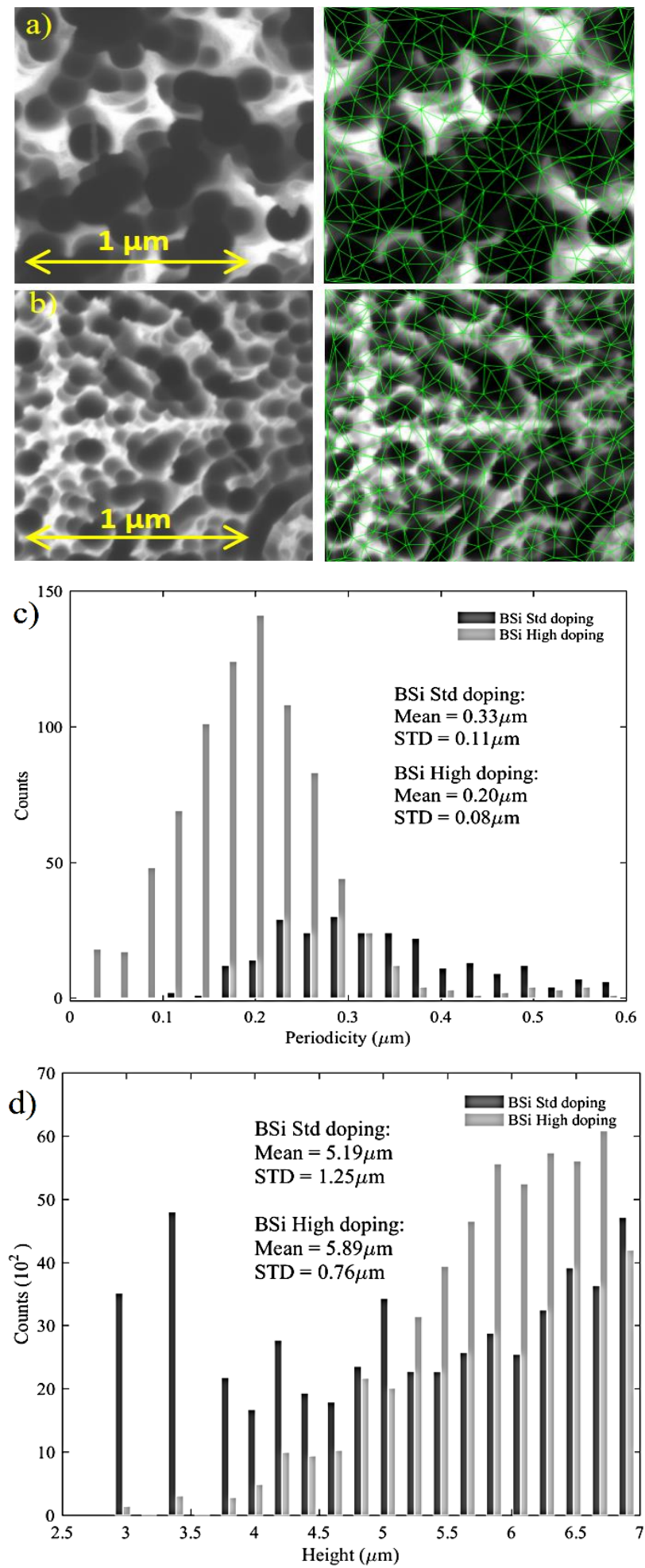

Figure 4: Morphological analysis of $1.3 \times 1.3{\mu m^{2}}^{2} B S i$ samples obtained from top-view SEM images. (a) SEM and model images of BSi etched on standard substrate. (b) SEM and model images of BSi etched on a highly doped substrate. (c) Histogram of etched hole's periodicity and (d) histogram of structure's height for both samples. All these data demonstrate an increase in the number of nanostructures and a decrease of their mean periodicity. 
(DRIE) [17, 18] have been accounted for. Plasma etching utilizing DRIE is a surely understood procedure to acquire black silicon surfaces of low reflectance. The DRIE depends on inductively coupled plasma (ICP) of sulphur hexafluoride (SF6) and permits anisotropic etching of silicon and this method is genuinely basic, fast, and controllable. While the purported 'Bosch' strategy is the most commonly used procedure in DRIE predominantly, we have used the cryogenic procedure to obtain black silicon surfaces, in this work [7]. The fixed parameters for all examples are ICP intensity of $1000 \mathrm{~W}$, gas weight of $1.5 \mathrm{~Pa}$, and SF6 gas stream rate of $200 \mathrm{sccm}^{4}$. The samples were delivered on single side polished (100)-oriented single crystalline silicon wafers of 4 in. breadth, having $n$-type standard low doping corresponding to a doping level of $2 \times 10^{18} \mathrm{~cm}^{-3}$ and for high doping, a doping level of $4.5083 \times 10^{19} \mathrm{~cm}^{-3}$, is considered on which several square samples of $1 \mathrm{~cm}^{2}$ have been prepared.

\subsection{Statistics Generation from SEM Images}

SEM grey-scale analysis is a reconstruction technique from which a three-dimensional model that represents the surface topography of BSi samples can be obtained. This method consists of inferring the structures' height based on the greyscale level of a top-view SEM image, using the known height values of the SEM brightest and darkest grey-level as bounds of an interpolating range. While other non-destructive methods of reconstruction such as the ones based on multiple images taken at different viewing angles have been demonstrated, they require repositioning the sample at different viewing angles and robust algorithms to detect the same features in the different images [19]. SEM grey-scale analysis has been effectively used in the reconstruction of BSi samples [20]; however, there are some limitations of the maximum hole depth detectable that are due to the limited SEM dynamical range. Therefore, while the SEM grey-scale analysis provides accurate topography information, it is limited to BSi samples with small or medium aspect ratios. In this work, from top- and side-view SEM pictures, the mean values of periodicity and height of the BSi structures have been extracted by grey-level analysis using MATLAB: all the centres of holes have been identified and each pixel corresponds to a height.

\subsection{Numerical Methods}

In the infrared region, the specific Drude model [21], which describes the frequency-dependent dielectric function of silicon, has been employed with more advanced models [22]. Thus, in the various modelling techniques involved in this work such as Rigorous Wave Coupled Analysis(RCWA), FEM (Finite Element Mesh Analysis), the equations of Drude formalism have been employed to construct the representative models for simulations

\section{Results}

Figure 1 shows the spectral responses of two BSi samples of high and standard low doping. The effects of high doping on the bulk material dielectric permittivity and on the BSi geometrical features at the nano-scale, can be obviously noted, where, for the highly doped BSi sample, reflectance is kept lower than with the standard low doping until $15 \mu \mathrm{m}$. As a reminder, this figure also recalls the typical responses recorded in the visible range; as most of the time, such a curve shows an increase of reflectance at the highest wavelengths, which also suggests that absorption should deteriorate if we increase the wavelength further. But surprisingly, this does not happen in our results shown in Figure 1. On the contrary, one can see that reflectance is kept very low, below $1 \%$ up to $10 \mu \mathrm{m}$. For the highly doped silicon, reflectance remains even below $0.1 \%$ up to $7.5 \mu \mathrm{m}$ and then starts increasing up to $1 \%$ at $15 \mu \mathrm{m}$. These outstanding radiative properties cover a significant part of the useful spectrum of a thermal radiation source operating between room temperature and $600 \mathrm{~K}$. Indeed, considering the spectral distribution of the blackbody intensity given by Planck's function [23] and the fraction emitted in the spectral range covered by our experimental results between 1 and $25 \mu \mathrm{m}$, one can note that this fraction reaches $83 \%$ and $97 \%$ for a radiation source at $300 \mathrm{~K}$ and $600 \mathrm{~K}$, respectively [23]. Such small reflectance, thus high emissivity, in this specific wavelength range is of paramount importance for various applications. As a matter of fact, because of the large density of free carriers, heavily doped silicon is opaque in the mid-infrared. Therefore, such small reflectance is synonymous with high emissivity, according to Kirchhoff's law and energy conservation principle, which paves the way to efficient radiation sources. Figure 2 shows the zooming in on the performance of doped and undoped BSi, clearly illustrating the fact that till $15 \mu \mathrm{m}$, the highly doped sample has lower reflectance. Figure 3 shows the 3D reconstruction and side view SEM images of a $1.7 \mu \mathrm{m}^{2} \mathrm{BSi}$ samples for the two doping levels, all process parameters being identical otherwise. One can clearly observe a large increase of the BSi peak's density with the high doping and sharper peaks are observed. This is confirmed and quantified by statistical image processing based on the grey-level analysis shown in Figure 4: the number of etched holes has multiplied by more than a factor 3 , their mean-periodicity has decreased from $330 \mathrm{~nm}$ to $200 \mathrm{~nm}$, and the number of higher structures (above $5 \mu \mathrm{m}$ ) has also increased considerably. According to Kirchhoff's law of thermal radiation [24], spectral emissivity is equal to spectral absorptivity at a given temperature. Consequently, such small reflectance in this specific wavelength enables considering doped BSi as a potential effective and highly emissive infrared source for various kinds of sensing applications based on spectrophotometry.

\section{Conclusion}

In conclusion, we show, that the considerably high absorptivity of BSi can be extended to the spectral range of thermal radiation (up to $15 \mu \mathrm{m}$ ) by using highly doped silicon. This striking behavior is not ascribed to the high level of doping of the nanostructured surface. The exceptionally high absorptivity levels also translate into high emissivity of doped 
BSi. Such remarkable properties enable considering doped $\mathrm{BSi}$ as a propitious candidate for numerous applications, such as radiative cooling, broadband absorbers including bolometers as well as broadband infrared light sources based on black body emission.

\section{Acknowledgements}

This work was supported by the I-SITE FUTURE Initiative (reference ANR-16-IDEX-0003) in the frame of the project NANO-4-WATER.

\section{Literature}

1. Rephaeli, Eden, Aaswath Raman, and Shanhui Fan. "Ultrabroadband photonic structures to achieve highperformance daytime radiative cooling." Nano letters 13, no. 4 (2013): 1457-1461.

2. Kou, Jun-long, Zoila Jurado, Zhen Chen, Shanhui Fan, and Austin J. Minnich. "Daytime radiative cooling using nearblack infrared emitters." Acs Photonics 4, no. 3 (2017): 626630.

3. Steglich, Martin, Dennis Lehr, Stephan Ratzsch, Thomas Käsebier, Frank Schrempel, Ernst-Bernhard Kley, and Andreas Tünnermann. "An ultra-black silicon absorber." Laser \& Photonics Reviews 8, no. 2 (2014): L13-L17.

4. Ma, Shijun, Shuang Liu, Qinwei Xu, Junwen Xu, Rongguo $\mathrm{Lu}$, Yong Liu, and Zhiyong Zhong. "A theoretical study on the optical properties of black silicon." AIP Advances 8, no. 3 (2018): 035010.

5. Yu, Xin-Yue, Zhen-Hua Lv, Chun-Hao Li, Xiao Han, and Ji-Hong Zhao. "The optical and electrical properties of codoped black silicon textured by a femtosecond laser and its application to infrared light sensing." IEEE Sensors Journal 16, no. 13 (2016): 5227-5231.

6. Lv, Jian, Ting Zhang, Peng Zhang, Yingchun Zhao, and Shibin Li. "Review application of nanostructured black silicon." Nanoscale research letters 13, no. 1 (2018): 110.

7. Saab, David Abi, Philippe Basset, Matthew J. Pierotti, Matthew L. Trawick, and Dan E. Angelescu. "Static and dynamic aspects of black silicon formation." Physical review letters 113, no. 26 (2014): 265502.

8. Saab, D. Abi, Shermila Mostarshedi, Philippe Basset, Stéphane Protat, Dan Angelescu, and Elodie Richalot. "Effect of black silicon disordered structures distribution on its wideband reduced reflectance." Materials Research Express 1, no. 4 (2014): 045045.

9. Nguyen, K. N, P. Basset, F. Marty, Y. Leprince-Wang, and T. Bourouina. "On the optical and morphological properties of microstructured Black Silicon obtained by cryogenic-enhanced plasma reactive ion etching." Journal of Applied Physics 113, no. 19 (2013): 194903.

10. Ko, Byoungsu, Dasol Lee, Trevon Badloe, and Junsuk Rho. "Metamaterial-Based Radiative Cooling: Towards Energy-Free All-Day Cooling." Energies 12, no. 1 (2019): 89.

11. Branz, Howard M., Vernon E. Yost, Scott Ward, Kim M. Jones, Bobby To, and Paul Stradins. "Nanostructured black silicon and the optical reflectance of graded-density surfaces." Applied Physics Letters 94, no. 23 (2009): 231121.

12. Hench, John J., and Z. D. E. N. E. K. Strakoš. "The RCWA method-a case study with open questions and perspectives of algebraic computations." Electronic Transactions on Numerical Analysis 31 (2008): 331-357.

13. Gittleman, J. I., E. K. Sichel, H. W. Lehmann, and R. Widmer. "Textured silicon: a selective absorber for solar thermal conversion." Applied Physics Letters 35, no. 10 (1979): 742-744.

14. Craighead, H. G., R. E. Howard, and D. M. Tennant. "Textured thin-film Si solar selective absorbers using reactive ion etching." Applied Physics Letters 37, no. 7 (1980): 653-655.

15. Liu, Xiaogang, Paul R. Coxon, Marius Peters, Bram Hoex, Jacqueline M. Cole, and Derek J. Fray. "Black silicon: fabrication methods, properties and solar energy applications." Energy \& Environmental Science 7, no. 10 (2014): 3223-3263.

16. Schnell, Martin, Ralf Ludemann, and Sebastian Schaefer. "Plasma surface texturization for multicrystalline silicon solar cells." In Conference Record of the Twenty-Eighth IEEE Photovoltaic Specialists Conference-2000 (Cat. No. 00CH37036), pp. 367-370. IEEE, 2000.

17. Jansen, Henri, Meint de Boer, Rob Legtenberg, and Miko Elwenspoek. "The black silicon method: a universal method for determining the parameter setting of a fluorinebased reactive ion etcher in deep silicon trench etching with profile control." Journal of Micromechanics and Microengineering 5, no. 2 (1995): 115.

18. Jansen, Henri, Meint de Boer, Johannes Burger, Rob Legtenberg, and Miko Elwenspoek. "The black silicon method II: The effect of mask material and loading on the reactive ion etching of deep silicon trenches." Microelectronic engineering 27, no. 1-4 (1995): 475-480.

19. Stampfl, J., S. Scherer, M. Gruber, and O. Kolednik. "Reconstruction of surface topographies by scanning electron microscopy for application in fracture research." Applied Physics A 63, no. 4 (1996): 341-346.

20. Zhu, Fu-Yun, Qi-Qi Wang, Xiao-Sheng Zhang, Wei Hu, Xin Zhao, and Hai-Xia Zhang. "3D nanostructure reconstruction based on the SEM imaging principle, and applications." Nanotechnology 25, no. 18 (2014): 185705.

21. Basu, Soumyadipta, and Mathieu Francoeur. "Near-field radiative transfer based thermal rectification using doped silicon." Applied Physics Letters 98, no. 11 (2011): 113106.

22. Marquier, François, Karl Joulain, J-P. Mulet, Rémi Carminati, J-J. Greffet, and Y. Chen. "Coherent spontaneous emission of light by thermal sources." Physical Review B 69, no. 15 (2004): 155412.

23. Howell, John R., M. Pinar Menguc, and Robert Siegel. Thermal radiation heat transfer. CRC press, 2015.

24. Howell, John R., M. Pinar Menguc, and Robert Siegel. Thermal radiation heat transfer. CRC press, 2015. 